Check for updates

Cite this: Nanoscale Adv., 2019, 1, 3372

\title{
A low Schottky barrier height and transport mechanism in gold-graphene-silicon (001) heterojunctions $\dagger$
}

Received 21st June 2019 Accepted 25th July 2019

DOI: $10.1039 / c 9 n a 00393 b$

\author{
Jules Courtin, ${ }^{\text {ab }}$ Sylvain Le Gall, ${ }^{c}$ Pascal Chrétien, ${ }^{c}$ Alain Moréac, ${ }^{\text {ab }}$ Gabriel Delhaye, ${ }^{\text {ab }}$ \\ Bruno Lépine, ${ }^{\text {ab }}$ Sylvain Tricot, ${ }^{a b}$ Pascal Turban, ${ }^{a b}$ Philippe Schieffer ${ }^{\text {ab }}$ \\ and Jean-Christophe Le Breton (D) *ab
}

rsc.li/nanoscale-advances

The interface resistance at metal/semiconductor junctions has been a key issue for decades. The control of this resistance is dependent on the possibility to tune the Schottky barrier height. However, Fermi level pinning in these systems forbids a total control over interface resistance. The introduction of $2 \mathrm{D}$ crystals between semiconductor surfaces and metals may be an interesting route towards this goal. In this work, we study the influence of the introduction of a graphene monolayer between a metal and silicon on the Schottky barrier height. We used X-ray photoemission spectroscopy to rule out the presence of oxides at the interface, the absence of pinning of the Fermi level and the strong reduction of the Schottky barrier height. We then performed a multiscale transport analysis to determine the transport mechanism. The consistency in the measured barrier height at different scales confirms the good quality of our junctions and the role of graphene in the drastic reduction of the barrier height.

\section{Introduction}

Metal/Si (M/Si) junctions are one of the most important interfaces for Si-based devices. Drastic reduction of the contact resistivity at $\mathrm{M} / \mathrm{Si}$ interfaces is necessary for next generation CMOS technology. The contact resistivity is directly determined by the height and width of the Schottky barrier formed at the $\mathrm{M}$ / $\mathrm{Si}$ interface. For n-type silicon, the reduction of interface resistance can be achieved by the reduction of the barrier width i.e. by the reduction of the space charge region width via heavy

${ }^{a}$ Univ. Rennes, CNRS, IPR (Institut de Physique de Rennes), UMR 6251, F-35000 Rennes, France. E-mail: jean-christophe.lebreton@univ-rennes.fr

${ }^{b}$ Département Matériaux et Nanosciences, Institut de Physique de Rennes, UMR 6251, CNRS, Université de Rennes 1, Campus de Beaulieu, Bât 11E, 35042 Rennes cedex, France

${ }^{c}$ Group of Electrical Engineering Paris (GeePs), CNRS, CentraleSupélec, Univ. Paris Sud, Sorbonne Université, CEDEX, 11 rue Joliot-Curie, 91192 Gif-sur-Yvette, France

$\dagger$ Electronic supplementary information (ESI) available: S1 - Graphene transfer protocol; S2 - Schottky barrier height XPS determination; S3 - Raman spectrum of gold protected graphene; S4 - Optical image, AFM image and resistance histograms of the areas under study. See DOI: 10.1039/c9na00393b doping. However, as devices are scaled down, leakage current and dopant profile control becomes increasingly difficult. Moreover, doping techniques such as implantation and diffusion require high temperatures in the $1000^{\circ} \mathrm{C}$ range which may be incompatible with some processes like MEMS integration. $\mathrm{Au}-\mathrm{Sb}$ alloys may form ohmic contacts with $\mathrm{Si}$ at a lower temperature of around $360{ }^{\circ} \mathrm{C}$.

For the Schottky barrier height $(\mathrm{SBH})$, reduction is more difficult because of the pinning of the Fermi level (FL) at approximately midgap in $\mathrm{Si}^{1}{ }^{1}$ Attempts to alleviate FL pinning in semiconductors have been made in the past by the introduction of a thin insulating barrier ${ }^{2,3}$ or surface passivation. ${ }^{4} \mathrm{~A}$ new and promising route towards this goal is the introduction of a $2 \mathrm{D}$ crystal between $\mathrm{M}$ and Si. As they are ultimately thin, $2 \mathrm{D}$ crystals would reduce the resistance increase due to insulator barriers and tune the work function., ${ }^{5,6}$ Graphene $(G)$, a one-atom-thick layer of carbon in a honeycomb crystal lattice, avoids the formation of undesired interface states and/ or metal induced states at the Si surface..$^{7,8}$ Moreover, the work function of graphene can be modulated by doping with various metals. ${ }^{9,10}$ Several experimental results show that, unlike other metals, the Fermi level pinning can be alleviated with graphene on Si. ${ }^{11,12}$ The literature on $\mathrm{M} / \mathrm{G} / \mathrm{Si}$ shows a variety of results. When heavily doped $\mathrm{Si}$ is used, the G layer acts as an almost transparent tunnel barrier which makes it a promising candidate for spin filtering for example. ${ }^{13-15}$ For lower doped $\mathrm{Si}$, a reduced interface resistance or $\mathrm{SBH}$ has been observed for $\mathrm{M} / \mathrm{G} / \mathrm{Si}$ compared to $\mathrm{M} / \mathrm{Si}^{\mathbf{5 , 1 1 , 1 6}}$ On the other hand, other groups report no influence of the $\mathrm{G}$ layer at all but with a large variation between the measured $\mathrm{SBHs}^{7,17}$ for similar stacks. Similar to what can be found for G/Si Schottky contacts, a number of different characterisation techniques, transport models and sample preparation methods make the comparison between different results difficult. For that reason, we present here a multiscale analysis of a $\mathrm{Au} / \mathrm{G} / \mathrm{Si}: \mathrm{H}$ junction studied with different techniques in order to give a consistent description of the band alignments and determine the transport mechanism. 
We used X-ray photoemission spectroscopy (XPS) to study the formation of $\mathrm{Au} / \mathrm{G} / \mathrm{Si}$ contacts. Core level peak positions were used to determine the energy band alignment. Capacitance-voltage $(\mathrm{C}-\mathrm{V})$ and current-voltage $(J-V)$ measurements and conductive-probe atomic force microscopy (CP-AFM) were then used to determine the apparent electronic SBH, transport mechanism and contact homogeneity. Using these multiscale techniques, we demonstrate the absence of FL pinning and strong reduction of the SBH induced by the insertion of monolayer graphene between $\mathrm{Au}$ and hydrogen-passivated silicon (Si:H).

\section{Experimental details}

Single layer graphene realized by chemical vapor deposition on copper was purchased from Graphenea $\odot$. A $200 \mathrm{~nm}$ polymethyl methacrylate (PMMA) layer was then deposited on top. After PMMA coating, copper was etched in a $\mathrm{FeCl}_{3}$ solution until no metallic copper could be seen and the floating PMMA/graphene was rinsed several times in deionized water. Graphene was then transferred onto a Si substrate with a resistivity of $1-10 \Omega \mathrm{cm}$. Si was degreased in acetone and isopropyl alcohol followed by 30 minutes of UV/ozone cleaning leading to the formation of a thin $\mathrm{SiO}_{2}$ layer of about $1 \mathrm{~nm}$ (see the ESI $\dagger$ ). The floating PMMA/ graphene was deposited onto the oxidized silicon and left to dry for 30 minutes at $50{ }^{\circ} \mathrm{C}$ followed by 15 minutes at $135{ }^{\circ} \mathrm{C}$. After removal of PMMA in acetone, the samples were introduced into an Ultra High Vacuum (UHV) chamber where they were annealed at $300{ }^{\circ} \mathrm{C}$ for 3 hours to remove resist residues. The samples were then taken out of the UHV chamber for a final oxide etch in $2 \%$ hydrofluoric acid (HF) before re-introduction into the UHV chamber. Au was then sequentially deposited at room temperature by molecular beam epitaxy with an effusion cell at a rate of $0.2-0.4 \AA \mathrm{min}^{-1}$ for submonolayer thicknesses and 1-2 $\AA \min ^{-1}$ otherwise. The Si $2 \mathrm{p}$ and C 1 s core levels were monitored as a function of gold thickness. Metal deposition and photoemission measurements were performed in a multichamber UHV system with base pressures below $2 \times 10^{-10}$ mbar. XPS measurements at normal emission were performed at room temperature using a $\mathrm{Mg} \mathrm{K} \alpha(1253.6 \mathrm{eV})$ source. The kinetic energy of the emitted electrons has been measured by employing a hemispherical analyzer (Omicron EA125) with a five channel detection system. The total energy resolution was $0.80 \mathrm{eV}$. The $\mathrm{Au} 4 \mathrm{f}_{7 / 2}$ peak position from a thick gold sample, assumed to be $84.00 \mathrm{eV} \mathrm{BE},{ }^{18}$ was taken as a reference in order to position the Fermi level (FL). For the determination of the SBHs and Si band bending, we assumed an energy difference of 98.74 $\mathrm{eV}$ between the valence band maximum of $\mathrm{Si}$ and the $\mathrm{Si} 2 \mathrm{p}_{3 / 2}$ core level (ref. 19) and a room temperature bandgap energy of $1.12 \mathrm{eV}$ for Si. For current-voltage and capacitance-voltage transport measurements, the same procedure was used for graphene transfer. The Si substrates were heavily doped on the back side prior to $\mathrm{G}$ transfer in order to make good ohmic contacts. After HF etch and introduction into a UHV, $30 \mathrm{~nm}$ thick metal contacts with diameters ranging from 150 to $500 \mu \mathrm{m}$ were deposited under a shadow mask. Graphene between the metal contacts was then oxidized with a UV/ozone step.
Transport measurements were carried out with a Keithley DC current sourcemeter. The capacitance-voltage $(\mathrm{C}-\mathrm{V})$ measurements were carried out using a HP4284 LCR meter with a sinusoidal voltage amplitude of $20 \mathrm{mV}$ and a frequency between 20 and $1 \mathrm{MHz}$. The electrical local characterization of the sample has been performed using a Bruker NanoScope 8.0 atomic force microscope (AFM) equipped with a specific electrical measurement module called "Resiscope" ${ }^{20}$ The method combines the nanometric scale resolution of the AFM and the real time electrical measurement of the ResiScope allowing resistance measurements over a ten decade range $\left(10^{4}\right.$ to $\left.10^{14} \Omega\right)$ under the dynamic conditions of AFM imaging. In this experimental configuration, the AFM $\mathrm{p}^{++-d i a m o n d}$ conductive tip scans over the surface of the sample in contact mode and under a controlled and constant normal force. Thanks to the polarization of the sample, the ResiScope analyzes the current across the tip in order to give, for each point of the surface scanned, a value of resistance. So, it is possible to build point by point a map of local electrical resistance parallel to a topography map. ${ }^{21}$

\section{Results and discussion}

Fig. 1a shows the experimental spectrum of the $\mathrm{C}$ 1s core level for single layer graphene (G) on n-type $\mathrm{Si}(001): \mathrm{H}$. We introduced four components to obtain a reasonable fit of the experimental curve. The main component located at $284.71 \pm 0.05 \mathrm{eV}$ binding energy ( $\mathrm{BE}$ ) corresponds to $\mathrm{C}-\mathrm{C} \mathrm{sp}^{2}$ bonds. A component located at $0.7 \mathrm{eV}$ higher $\mathrm{BE}$ is attributed to $\mathrm{sp}^{3}$ carbons. ${ }^{22,23}$ Two more components at $2.4 \mathrm{eV}$ and $4.2 \mathrm{eV}$ higher $\mathrm{BE}$ ( $\mathrm{C} 1$ and $\mathrm{C} 2$ in the figure) are assigned to contributions of carbon atoms from PMMA residues, ${ }^{23,24}$ and the intensity of these components is reduced by UHV annealing. The doping concentration in graphene can be determined by the difference in the $\mathrm{C} 1 \mathrm{~s}-\mathrm{sp}^{2}$ peak position between monolayer graphene and Highly Oriented Pyrolytic Graphite (HOPG). Lin et al. ${ }^{25}$ observed that the C $1 \mathrm{~s}$ core level of undoped suspended graphene is shifted by $0.4 \mathrm{eV}$ toward higher BE compared to the bulk component of multilayer graphene (MLG). Assuming a similar C 1s BE between freshly pealed HOPG and the bulk component of MLG, it was found that the Dirac point is located at $0.25 \pm 0.05 \mathrm{eV}$ above the FL for $\mathrm{G}$ transferred on $\mathrm{Si}(001): \mathrm{H}$ surfaces indicating p-type doping of graphene with a typical hole carrier density of $4.6 \times$ $10^{12} \mathrm{~cm}^{-2}$.

As gold is sequentially deposited on the G/Si sample, the position of the $\mathrm{C} 1 \mathrm{~s}$ core level gradually shifts towards lower BE. This is assigned to gradual p-doping induced by charge transfer from Si to Au. ${ }^{9}$ Fig. 2 shows the energy difference between the Fermi level and the Dirac point in graphene as well as the SBH of $\mathrm{Si}$ as a function of Au coverage. The Fermi level shifts from $-0.25 \pm 0.05 \mathrm{eV}$ from the Dirac point before to $-0.38 \pm 0.05 \mathrm{eV}$ after a $3 \mathrm{~nm}$ thick Au layer deposition (the negative sign indicates p-type doping).

The top curve in Fig. 1b shows the Si 2p core level for G/Si:H. An almost complete removal of $\mathrm{SiO}_{2}$ through the graphene layer is observed after the HF treatment. When present, the remaining silicon oxide thickness deduced from the ratio of $\mathrm{Si}^{4+}$ 

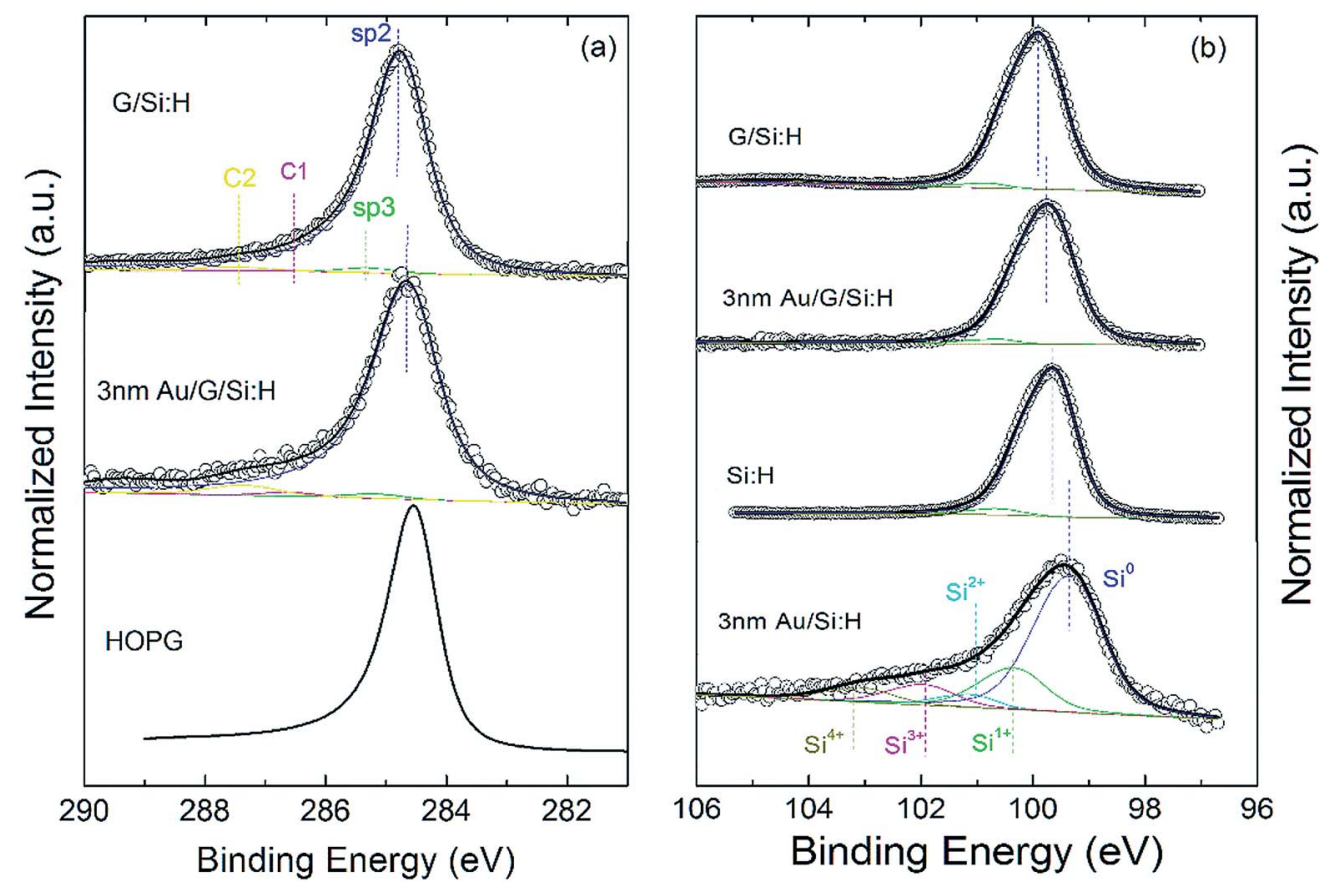

Fig. 1 (a) C 1s core level spectra of G/Si:H before and after $3 \mathrm{~nm}$ Au deposition. The $\mathrm{C}$ 1s level of HOPG is also shown. (b) Si 2p core level spectra of Si:H and G/Si:H before and after $3 \mathrm{~nm}$ Au deposition.

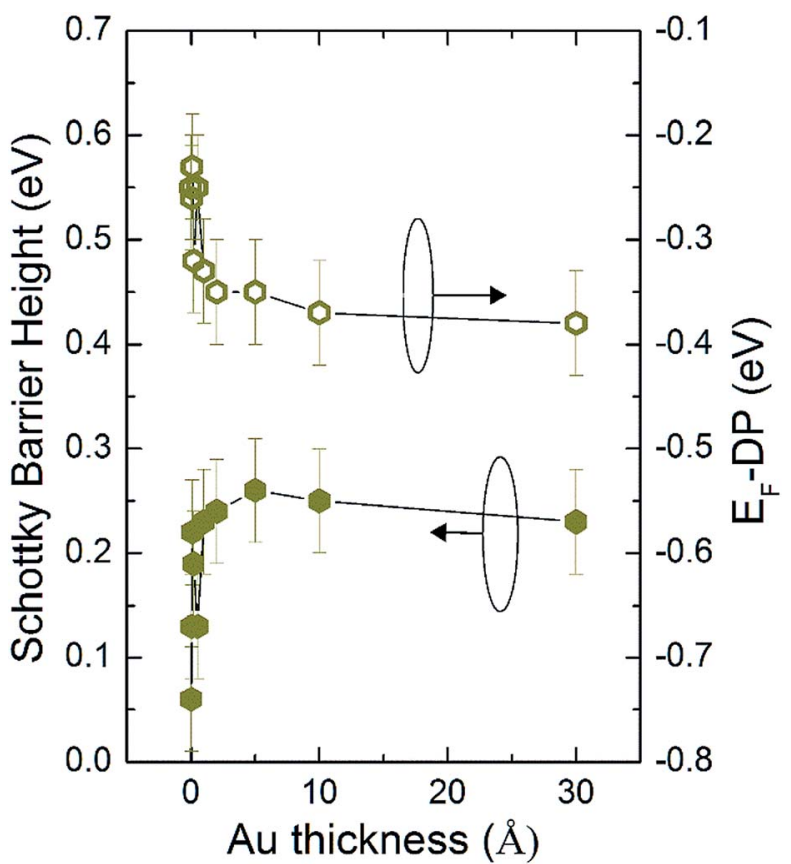

Fig. 2 Schottky barrier height of Si and energy difference between the Dirac point and the Fermi level in the graphene layer as a function of Au coverage.

(located at $3.75 \mathrm{eV}$ from the $\mathrm{Si} 2 \mathrm{p}_{3 / 2}$ peak) to Si-bulk components $^{26}$ is estimated to be less than about $0.1 \mathrm{~nm}$. The $\mathrm{BE}$ position of the Si $2 \mathrm{p}_{3 / 2}$ core level leads to a $\mathrm{SBH}$ of $0.06 \pm 0.05 \mathrm{eV}$ (see the $\mathrm{ESI} \dagger$ for $\mathrm{SBH}$ determination). For the bare Si:H surface the $\mathrm{BE}$ position corresponds to a SBH of $0.30 \pm 0.05 \mathrm{eV}$ for a similar doping concentration.
A large discrepancy can be found in the literature for the $\mathrm{G} / \mathrm{Si}$ $\mathrm{SBH}$ (from 0.32 to $0.89 \mathrm{eV}$ (ref. 11 and 27-35)). Nevertheless, the SBH of our SLG/Si:H junctions determined by XPS is very low. Most results from the literature showing a higher SBH were obtained by transport measurements. In this case, hot spots due to defects or ripples may dominate the electrical transport through the junction and may reflect the electrical properties of only a small proportion of the junction surface. Other parameters such as the efficiency of hydrogen passivation, the residual silicon oxide under the graphene sheet or the graphene transfer quality may differ from sample to sample. In contrast, the photoemission experiment gives an averaged value of the $\mathrm{SBH}$ at a macroscopic scale and is widely insensitive to microscopic defects. Nevertheless, other reasons may explain the difference between our results and those of the literature as we will see in the next paragraph.

In the simple Schottky-Mott model, the $\mathrm{SBH} \phi_{\mathrm{B}}$ is the difference between the work function of graphene $W_{\mathrm{G}}$ and the electronic affinity of the SC $\chi_{\mathrm{Si}}$ :

$$
\phi_{\mathrm{B}}=W_{\mathrm{G}}-\chi_{\mathrm{Si}}
$$

The commonly used work function of graphene is $4.54 \mathrm{eV}$ and the electronic affinity of $\mathrm{Si}$ is $4.05 \mathrm{eV}$. This would give rise to a $\phi_{\mathrm{B}}$ of $0.49 \mathrm{eV}$, much larger than our measured SBH of $0.06 \mathrm{eV}$. However, for CVD graphene deposited via the PMMA assisted method, the work function was measured by Lin et al. to be $W_{\mathrm{G}}$ $=4.3 \mathrm{eV}$, lower than the commonly used $4.54 \mathrm{eV}$ because of resist contamination and structural defects induced during wet transfer. $^{36}$ This would lead to an expected SBH of $0.25 \mathrm{eV}$. 
Moreover, a reduction of the $\mathrm{SBH}$ is expected for $\mathrm{Si}: \mathrm{H}$ because of an electric dipole $\mathrm{H}^{-\delta q}-\mathrm{Si}^{+\delta q}$ at the Si surface. A reduction of $0.21 \mathrm{eV}$ was measured for $\mathrm{Pb} / n-\mathrm{Si}: \mathrm{H}$ compared to $\mathrm{Pb} / n-\mathrm{Si}^{37} \mathrm{~A}$ similar $0.21 \mathrm{eV}$ reduction would lead to a SBH of $0.04 \mathrm{eV}$. This value is very close to the $\mathrm{SBH}$ we measure here. Our particularly low barrier may therefore be explained by the low work function of wet transferred CVD-graphene and the surface dipole. A better graphene quality and the absence of hydrogen passivation are expected to lead to a larger $\mathrm{SBH}$.

With Au sequentially deposited, the Schottky barrier forms and the position of the bulk Si 2 p component gradually shifts towards lower BE. For the uncovered sample, a $0.39 \mathrm{eV}$ shift of the Si 2 p core level is observed after $3 \mathrm{~nm}$ Au deposition giving rise to a final $\mathrm{SBH}$ of $0.66 \pm 0.05 \mathrm{eV}$, slightly lower than the 0.75 $\pm 0.04 \mathrm{eV}$ measured by Grupp and Taleb-Ibrahimi. ${ }^{38}$ Several Si $2 \mathrm{p}$ components are needed to fit the Si $2 \mathrm{p}$ peak for the $\mathrm{Au} / \mathrm{Si}$ sample. These peaks are attributed to surface components induced by $\mathrm{Si}$ diffusion through $\mathrm{Au}$ and subsequent oxidation. ${ }^{39}$

For the graphene-covered sample, the position of the bulk Si $2 \mathrm{p}$ component gradually shifts towards lower binding energy after $3 \mathrm{~nm}$ Au deposition giving rise to a final SBH of $0.23 \pm 0.05$ $\mathrm{eV}$, as shown in Fig. 2. This SBH is stable after $5 \AA$ Au deposition. No reaction component is needed to fit the $\mathrm{Si} 2 \mathrm{p}$ core level. This result indicates that graphene is an efficient barrier against metal diffusion as already pointed out by several groups., ${ }^{7,8,40}$ The evolution of the $\mathrm{SBH}$ and doping concentration in the graphene layer as a function of $\mathrm{Au}$ coverage is presented in Fig. 2. As the FL in graphene shows a downshift of $0.13 \pm 0.05$ $\mathrm{eV}$ from the Dirac point with increasing gold coverage, the $\mathrm{SBH}$ shows an almost similar upshift of $0.17 \pm 0.05 \mathrm{eV}$. These variations demonstrate the absence of pinning at the $\mathrm{G} / \mathrm{Si}: \mathrm{H}$ interface.

Capacitance-voltage $(\mathrm{C}-\mathrm{V})$ and current density-voltage $(J-V)$ measurements were carried out to confirm the XPS results and determine the transport mechanism. A voltage bias across the Schottky barrier is able to modulate the depletion region width $w$ and to change the charge located at the junction, making it behave as a parallel plate capacitor. When a small AC voltage $\delta V$ is added to the reverse DC bias $(V<0)$, the junction shows a capacitive behavior with capacitance per unit area expressed as $C=\left|\frac{\delta Q_{\mathrm{d}}}{\delta V}\right|=\frac{\varepsilon_{\mathrm{s}}}{w}=\sqrt{\frac{q \varepsilon_{\mathrm{s}} N_{\mathrm{D}}}{2\left(\varphi_{\mathrm{i}}-V-\frac{k_{\mathrm{b}} T}{q}\right)}}$ where $\varepsilon_{\mathrm{s}}$ is the dielectric constant of $\mathrm{Si}, N_{\mathrm{D}}$ is the doping concentration, $\varphi_{\mathrm{i}}$ is the surface potential, $V$ is the applied voltage, $q$ is the electronic charge, $k_{\mathrm{B}}$ is the Boltzmann constant and $T$ is the temperature. A plot of $1 / C^{2}$ versus $V$ should therefore be a straight line with the slope being $2 / q \varepsilon_{\mathrm{S}} N_{\mathrm{D}}$ and the intercept being proportional to $\varphi_{\mathrm{i}}-k T / q$. The built in potential $\varphi_{\mathrm{i}}$ is related to the $\mathrm{SBH}$ as $\phi_{\mathrm{B}}=\varphi_{\mathrm{i}}+k T \ln \left(\frac{N_{\mathrm{C}}}{N_{\mathrm{D}}}\right)$

Fig. 3a shows the $1 / C^{2}$ versus $V$ plot obtained at $1 \mathrm{MHz}$ for different temperatures. The curves show straight parallel lines for $V<0$. This is indicative of good quality Schottky junctions with a low density of interface states ${ }^{\mathbf{4 1}}$ and a doping concentration independent of temperature. Fig. $3 \mathrm{~b}$ shows the $\mathrm{SBH} \phi_{\mathrm{B}}$ deduced from these measurements as a function of temperature from $0.30 \mathrm{eV}$ at $250 \mathrm{~K}$ down to $0.25 \mathrm{eV}$ at $330 \mathrm{~K}$. These results are in good agreement with the SBH deduced from XPS.

Fig. 4a shows the measured $I-V$ characteristics between 220 $\mathrm{K}$ and $320 \mathrm{~K}$. Non-linear rectifying behavior was observed for all the devices indicating thermionic emission (TE) dominated transport over the barrier. A significant increase in reverse current density was observed as the temperature raised from $220 \mathrm{~K}$ to $320 \mathrm{~K}$. This is consistent with the reduction of the effective barrier height observed from $\mathrm{C}-\mathrm{V}$ in Fig. $3 \mathrm{~b}$. The forward bias current density shows temperature dependence as well. The $I-V$ characteristic of a Au contact on Si outside the Gcovered area at $300 \mathrm{~K}$ is also shown for comparison.

The zero-bias Schottky barrier height, $\phi_{\mathrm{B}}^{0}$, and the ideality factor $n$ at room temperature (RT) can be extracted using (TE) theory according to the following equation:

$$
\begin{aligned}
J & =A^{* *} T^{2} \exp \left(\frac{-q \phi_{\mathrm{B}}^{0}}{k_{\mathrm{B}} T}\right)\left[\exp \left(\frac{q V}{n k_{\mathrm{B}} T}\right)-1\right] \\
& =J_{0}\left[\exp \left(\frac{q V}{n k_{\mathrm{B}} T}\right)-1\right]
\end{aligned}
$$

where $J$ is the current density, $q$ is the electronic charge, $k_{\mathrm{B}}$ is the Boltzmann constant, $T$ is the temperature, and $A^{* *}$ is the
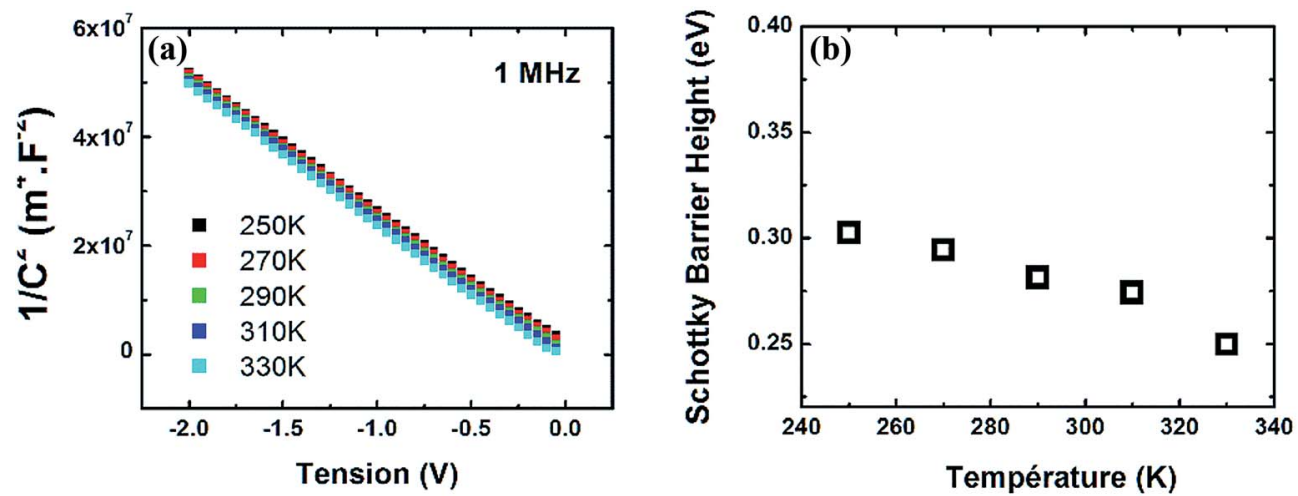

Fig. 3 (a) $1 / C^{2}$ vs. $V$ plot at $1 \mathrm{MHz}$ for a Au/G/Si:H junction between $250 \mathrm{~K}$ and $330 \mathrm{~K}$. (b) Schottky barrier height deduced from the capacitancevoltage measurements as a function of temperature. 

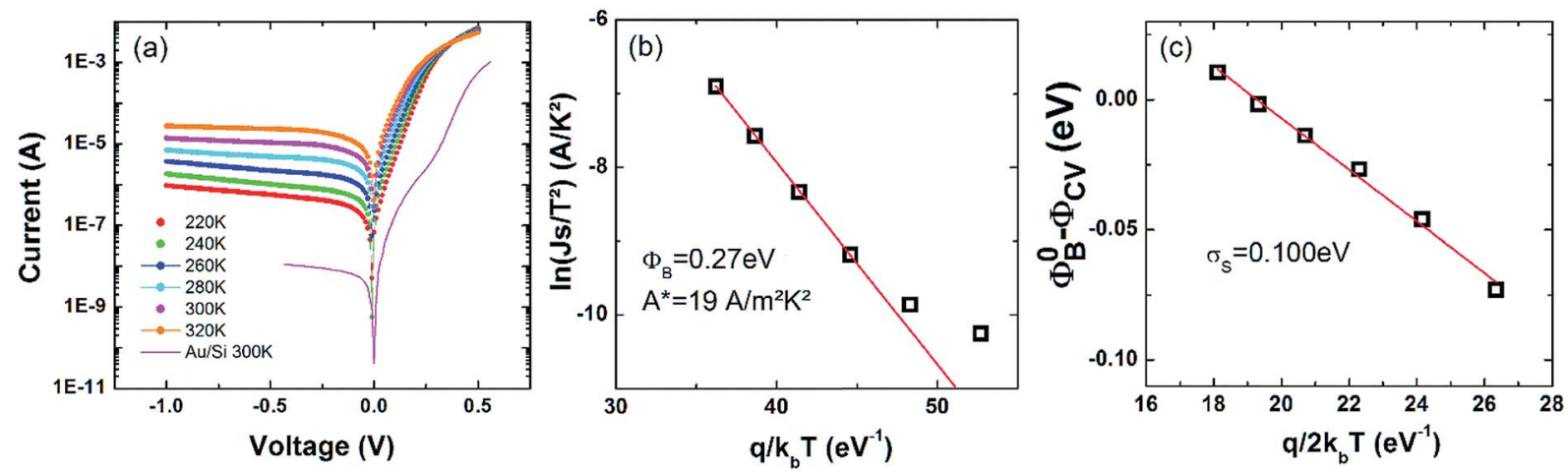

Fig. 4 (a) Current-voltage measurements between $220 \mathrm{~K}$ and $320 \mathrm{~K}$ for Au/G/Si:H. The room temperature $/-V$ characteristic of a Au/Si contact is also shown. (b) Richardson plot of the Au/G/Si:H junction and (c) determination of the standard deviation from the mean barrier height.

Richardson constant. $A^{* *}=1.12 \times 10^{6} \mathrm{~A} \mathrm{~m}^{-2} \mathrm{~K}^{-2}$ for n-type $\mathrm{Si}(100)$ for intimate metal/Si contacts. However, many parameters can influence the true $A^{* *}$ value such as barrier inhomogeneity, effective surface area, quality of the interface, etc. We should stress that the zero bias $\mathrm{SBH}$ deduced from $J-V$ measurements is strongly dependent on the choice of $A^{* *}$. It is therefore necessary to determine the effective Richardson constant with a plot of $\ln \left(J_{\mathrm{s}} / T^{2}\right)$ versus $q / k_{\mathrm{B}} T$ as shown in Fig. $4 \mathrm{~b}$, where $J_{\mathrm{s}}$ is the reverse saturation current density.

This plot is well fitted with a straight line between 260 and $320 \mathrm{~K}$. The deviation from linearity below $260 \mathrm{~K}$ indicates a temperature dependent barrier height. The slope of such a plot gives $\phi_{\mathrm{B}}=0.27 \mathrm{eV}$, the effective $\mathrm{SBH}$ which is consistent with the barrier height of $0.23 \mathrm{eV}$ measured by XPS and $0.28 \mathrm{eV}$ by $\mathrm{C}-\mathrm{V}$ at room temperature. The intercept gives an effective Richardson constant $A^{* *}$ of $19 \mathrm{~A} \mathrm{~m}^{-2} \mathrm{~K}^{-2}, 5$ orders of magnitude lower than the expected $A^{* *}$ for $\mathrm{Si}(001)$. Most reports on $\mathrm{G} /$ Si Schottky barriers show similarly low Richardson constants. ${ }^{\mathbf{4 2 - 4 5}}$ This was attributed to the finite density of states in graphene and 2D massless Dirac fermion characteristics of its carriers ${ }^{29}$ or to inhomogeneous SBHs due to potential fluctuations at the interface.
For the latter case, these fluctuations are described through the Werner model ${ }^{46}$ which correlates the mean $\phi_{\mathrm{CV}}(T)$ and the apparent barrier $\phi_{\mathrm{B}}^{0}(T)$ :

$$
\phi_{\mathrm{B}}^{0}(T)=\phi_{\mathrm{CV}}(T)-\frac{q \sigma_{\mathrm{s}}^{2}}{2 k_{\mathrm{B}} T}
$$

where $\sigma_{\mathrm{s}}$ is the standard deviation from the mean barrier height, $\phi_{\mathrm{CV}}(T)$ is the mean barrier height i.e. the SBH deduced from CV measurements and $\phi_{\mathrm{B}}^{\mathrm{O}}(T)$ is the apparent SBH calculated from eqn (1) with the $A^{* *}$ deduced from the Richardson plot. $\phi_{\mathrm{B}}^{\mathrm{O}}(T)-\phi_{\mathrm{CV}}(T)$ is plotted as shown in Fig. $4 \mathrm{c}$ as a function of $\frac{q}{2 k_{\mathrm{B}} T}$ and gives a standard deviation of $\sigma_{\mathrm{s}}=100 \mathrm{meV}$.

Experimental confirmation of this model was realized with conductive-probe atomic force microscopy (CP-AFM). Fig. 5a shows an AFM scan of the edge of a $30 \mathrm{~nm} \mathrm{Au}$ contact at the interface between uncovered and G-covered Si. CP-AFM mapping of the same area is shown in Fig. 5b. Four different interfaces can be seen in this picture at a bias voltage of $-2 \mathrm{~V}: \mathrm{G} /$ $\mathrm{Si}, \mathrm{Si}, \mathrm{Au} / \mathrm{G} / \mathrm{Si}$ and $\mathrm{Au} / \mathrm{Si}$. Note that the regions unprotected by gold were oxidized with a UV/ozone step. The $\mathrm{Au} / \mathrm{Si}$ contact shows a large homogeneous local resistance in the $10^{13} \Omega$ range

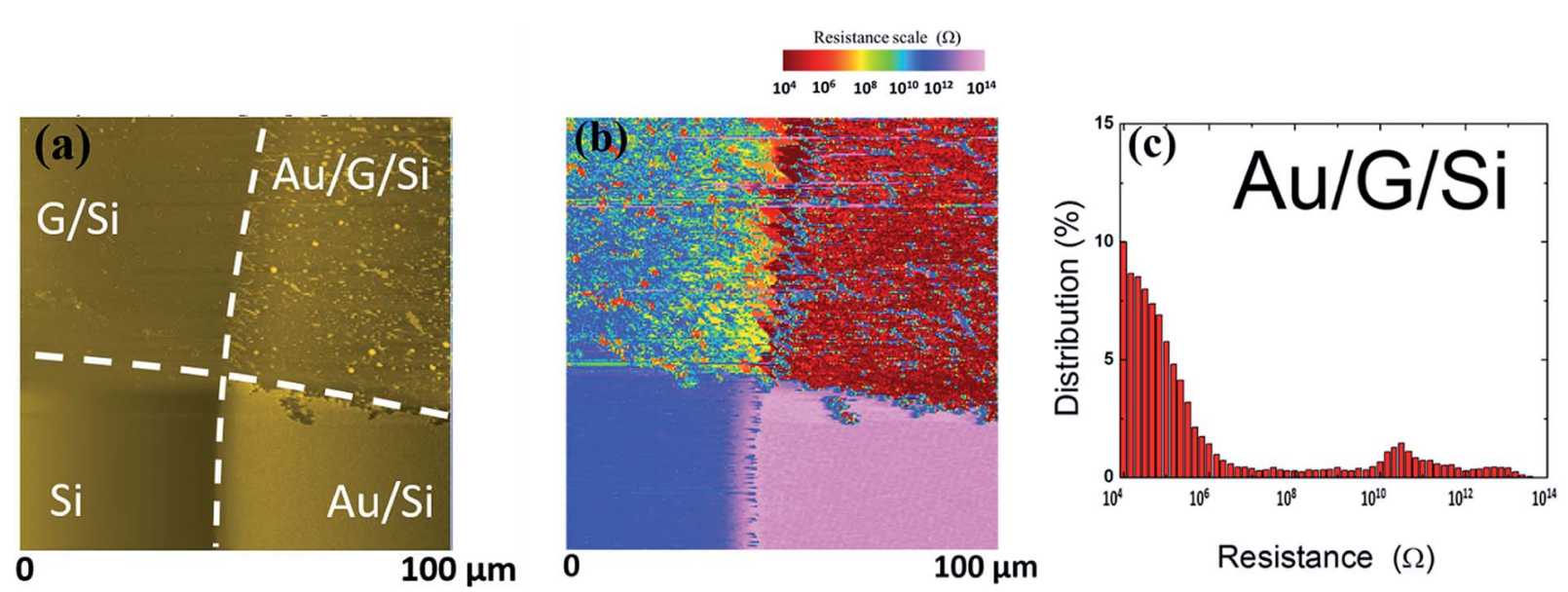

Fig. 5 (a) AFM close-up showing the four regions, (b) ResiScope mapping at the interface between uncovered and G covered Si under $30 \mathrm{~nm}$ Au, and (c) resistance distribution in the $\mathrm{Au} / \mathrm{G} / \mathrm{Si}$ area. 
(see the resistance histograms in ESI Fig. S3†) probably due to both the large SBH expected for Au/Si Schottky contacts and the diffusion of Si through the Au layer plus subsequent oxidation at the surface. When the graphene layer is present at the interface between $\mathrm{Au}$ and $\mathrm{Si}$, we observe a strong reduction of local resistance consistent with the low SBH observed by XPS, $\mathrm{C}-\mathrm{V}$ and $J-V$. Most of the surface shows a local resistance in the $10^{4}$ to $10^{6} \Omega$ range with small areas of larger resistance in the $10^{10} \Omega$ range. These local high resistive spots of $\sim 10^{10} \Omega$ are related to the topography and are therefore attributed to resist residues. If we consider the local resistance to be dominated by the $\mathrm{SBH}$ at the interface, these measurements confirm that the $\mathrm{Au} / \mathrm{G} / \mathrm{Si} \mathrm{SBH}$ is low and inhomogeneous with a Gaussian distribution centered at $10^{4} \Omega$, our detection limit.

\section{Conclusions}

We studied $\mathrm{Au} / \mathrm{G} / \mathrm{Si}$ heterojunctions by different techniques so as to determine their interface physico-chemistry, band alignments, homogeneity and electrical transport characteristics. We observed by XPS successful Si oxide removal after graphene transfer and an absence of intermixing between Si and Au. XPS was also used to determine the band alignments: for $\mathrm{G} / \mathrm{Si}: \mathrm{H}$, the $\mathrm{Si} \mathrm{SBH}$ is close to zero and the progressive increase of graphene p-doping by $\mathrm{Au}$ induces a similar progressive increase of the $\mathrm{Si}$ SBH which means that FL pinning was alleviated at the surface. Multiscale transport techniques confirmed the $\mathrm{SBH}$ value of $\mathrm{Au} /$ $\mathrm{G} / \mathrm{Si}: \mathrm{H}$ in the $0.25 \mathrm{eV}$ range at room temperature and identified thermionic emission as the dominant transport process. The Richardson constant was very low compared to the expected value for ideal Metal/Si Schottky junctions. This low value was attributed to an inhomogeneous SBH as confirmed by CP-AFM imaging. These results show that, in order to form a low resistance contact between Si and graphene, an appropriate choice of metal is necessary. This metal should induce n-type doping of the graphene sheet in order to further reduce the SBH on the Si side and to form a low resistance contact with graphene.

\section{Conflicts of interest}

The authors declare no competing financial interest.

\section{Acknowledgements}

The authors acknowledge Bruno Bêche for his help with AFM measurements and ANR grant ENSEMBLE for financial support. We thank the SIR platform of ScanMAT at Univ. Rennes 1 for technical support for the Raman measurements.

\section{References}

1 W. Mönch, J. Vac. Sci. Technol., B: Microelectron. Process. Phenom., 1988, 6, 1270.

2 Y. Zhou, M. Ogawa, X. Han and K. L. Wang, Appl. Phys. Lett., 2008, 93, 202105.

3 S. Gupta, P. Paramahans Manik, R. Kesh Mishra, A. Nainani, M. C. Abraham and S. Lodha, J. Appl. Phys., 2013, 113, 1-7.
4 M. Y. Ali and M. Tao, J. Appl. Phys., 2007, 101, 103708.

5 M. H. Lee, Y. Cho, K. E. Byun, K. W. Shin, S. G. Nam, C. Kim, H. Kim, S. A. Han, S. W. Kim, H. J. Shin and S. Park, Nano Lett., 2018, 18, 4878-4884.

6 S.-G. Nam, Y. Cho, M.-H. Lee, K. W. Shin, C. Kim, K. Yang, M. Jeong, H.-J. Shin and S. Park, 2D Mater., 2018, 5, 041004.

7 C. P. Y. Wong, T. J. H. Koek, Y. Liu, K. P. Loh, K. E. J. Goh, C. Troadec and C. A. Nijhuis, ACS Appl. Mater. Interfaces, 2014, 6, 20464-20472.

8 J. Hong, S. Lee, S. Lee, H. Han, C. Mahata, H.-W. Yeon, B. Koo, S.-I. Kim, T. Nam, K. Byun, B.-W. Min, Y.-W. Kim, H. Kim, Y.-C. Joo and T. Lee, Nanoscale, 2014, 6, 7503-7511.

9 G. Giovannetti, P. Khomyakov, G. Brocks, V. Karpan, J. van den Brink and P. Kelly, Phys. Rev. Lett., 2008, 101, 026803.

10 P. A. Khomyakov, G. Giovannetti, P. C. Rusu, G. Brocks, J. Van Den Brink and P. J. Kelly, Phys. Rev. B: Condens. Matter Mater. Phys., 2009, 79, 195425.

11 H. Yang, J. Heo, S. Park, H. J. Song, D. H. Seo, K.-E. Byun, P. Kim, I. Yoo, H.-J. Chung and K. Kim, Science, 2012, 336, 1140-1143.

12 K.-E. Byun, S. Park, H. Yang, H.-J. Chung, H. J. Song, J. Lee, D. H. Seo, J. Heo, D. Lee, H. J. Shin and Y. S. Woo, 2012 IEEE Nanotechnol. Mater. Devices Conf., 2012, pp. 63-66.

13 O. M. J. van't Erve, A. L. Friedman, E. Cobas, C. H. Li, a. T. Hanbicki, K. M. McCreary, J. T. Robinson and B. T. Jonker, J. Appl. Phys., 2013, 113, 17C502.

14 O. M. J. van't Erve, a L. Friedman, E. Cobas, C. H. Li, J. T. Robinson and B. T. Jonker, Nat. Nanotechnol., 2012, 7, 737-742.

15 X. Zhu, S. Lei, S. H. Tsai, X. Zhang, J. Liu, G. Yin, M. Tang, C. M. Torres, A. Navabi, Z. Jin, S. P. Tsai, H. Qasem, Y. Wang, R. Vajtai, R. K. Lake, P. M. Ajayan and K. L. Wang, Nano Lett., 2018, 18, 682-688.

16 K.-E. Byun, H.-J. Chung, J. Lee, H. Yang, H. J. Song, J. Heo, D. H. Seo, S. Park, S. W. Hwang, I. Yoo and K. Kim, Nano Lett., 2013, 13, 4001-4005.

17 I. Goykhman, U. Sassi, B. Desiatov, N. Mazurski, S. Milana, D. De Fazio, A. Eiden, J. Khurgin, J. Shappir, U. Levy and A. C. Ferrari, Nano Lett., 2016, 16, 3005-3013.

18 M. P. Seah, Surf. Interface Anal., 1989, 14, 488.

19 F. J. Himpsel, G. Hollinger and R. A. Pollak, Phys. Rev. B: Condens. Matter Mater. Phys., 1983, 28, 7014-7018.

20 O. Schneegans, P. Chrétien and F. Houze, Patents WO 2011/ 138738, 2011.

21 P. Narchi, J. Alvarez, P. Chrétien, G. Picardi, R. Cariou, M. Foldyna, P. Prod'homme, J. P. Kleider and P. R. i. Cabarrocas, Nanoscale Res. Lett., 2016, 11, 1-8.

22 A. Nikitin, L.-̊․ Näslund, Z. Zhang and A. Nilsson, Surf. Sci., 2008, 602, 2575-2580.

23 G. Cunge, D. Ferrah, C. Petit-Etienne, A. Davydova, H. Okuno, D. Kalita, V. Bouchiat and O. Renault, J. Appl. Phys., 2015, 118, 123302.

24 Y.-C. Lin, C.-C. Lu, C.-H. Yeh, C. Jin, K. Suenaga and P.-W. Chiu, Nano Lett., 2012, 12, 414-419.

25 C. Lin, H. Shiu, L. Chang, C. Chen, C. Chang and F. S. Chien, J. Phys. Chem. C, 2014, 118, 24898-24904. 
26 J. R. Shallenberger, D. a. Cole, S. W. Novak, R. L. Moore, M. J. Edgell, S. P. Smith, C. J. Hitzman, J. F. Kirchhoff, E. Principe, S. Biswas, R. J. Bleiler, W. Nieveen and K. Jones, 1998 Int. Conf. Ion Implant. Technol. Proc., (Cat. No. 98EX144), 1999, vol. 1, pp. 79-82.

27 S. Tongay, M. Lemaitre, X. Miao, B. Gila, B. R. Appleton and a. F. Hebard, Phys. Rev. X, 2012, 2, 011002.

28 S. Parui, R. Ruiter, P. J. Zomer, M. Wojtaszek, B. J. Van Wees and T. Banerjee, J. Appl. Phys., 2014, 116, 1-6.

29 D. Sinha and J. U. Lee, Nano Lett., 2014, 14, 4660-4664.

30 X. An, F. Liu, Y. J. Jung and S. Kar, Nano Lett., 2013, 13, 909916.

31 F. Liu and S. Kar, ACS Nano, 2014, 8, 10270-10279.

32 X. Miao, S. Tongay, M. K. Petterson, K. Berke, A. G. Rinzler, B. R. Appleton and A. F. Hebard, Nano Lett., 2012, 12, 27452750.

33 E. Shi, H. Li, L. Yang, L. Zhang, Z. Li, P. Li, Y. Shang, S. Wu, X. Li, J. Wei, K. Wang, H. Zhu, D. Wu, Y. Fang and A. Cao, Nano Lett., 2013, 13, 1776-1781.

34 H. Y. Kim, K. Lee, N. McEvoy, C. Yim and G. S. Duesberg, Nano Lett., 2013, 13, 2182-2188.
35 A. Singh, M. A. Uddin, T. Sudarshan and G. Koley, Small, 2014, 10, 1555-1565.

36 W.-H. Lin, T.-H. Chen, J.-K. Chang, J.-I. Taur, Y.-Y. Lo, W.-L. Lee, C.-S. Chang, W.-B. Su and C.-I. Wu, ACS Nano, 2014, 8, 1784-1791.

37 W. Mönch, Europhys. Lett., 1994, 27, 479-484.

38 C. Grupp and A. Taleb-Ibrahimi, Phys. Rev. B: Condens. Matter Mater. Phys., 1998, 57, 6258-6261.

39 A. Cros and P. Muret, Mater. Sci. Rep., 1992, 8, 271-367.

40 J. C. Le Breton, S. Tricot, G. Delhaye, B. Lépine, P. Turban and P. Schieffer, Appl. Phys. Lett., 2016, 109, 051601.

41 S. J. Fonash, J. Appl. Phys., 1982, 54, 1966-1975.

42 D. Tomer, S. Rajput, L. J. Hudy, C. H. Li and L. Li, Nanotechnology, 2015, 26, 215702.

43 S. Liang, W. Hu, A. Di Bartolomeo, S. Adam and L. K. Ang, IEEE Int. Electron Devices Meet., 2016, 14.4.1-14.4.4.

44 D. P. Sinha and J. U. Lee, Nano Lett., 2014, 14, 4660-4664.

45 A. Di Bartolomeo, F. Giubileo, G. Luongo, L. Iemmo, N. Martucciello, G. Niu, M. Fraschke, O. Skibitzki, T. Schroeder and G. Lupina, 2D Mater, 2017, 4, 015024.

46 J. H. Werner and H. H. Güttler, J. Appl. Phys., 1991, 69, 15221533. 